\title{
RESCAN: An actinic lensless microscope for defect inspection of EUV reticles
}

\author{
Iacopo Mochi*a, Patrick Helfenstein ${ }^{\mathrm{a}}$, Istvan Mohacsia, \\ Rajendran Rajeev ${ }^{\mathrm{a}}$, Shusuke Yoshitake ${ }^{\mathrm{b}}$, Yasin Ekinci ${ }^{\mathrm{a}}$

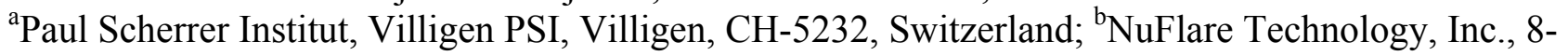 \\ 1 Shinsugita-cho, Isogo-ku, Yokohama 235-8522, Japan.
}

\begin{abstract}
Actinic mask defect inspection is an essential process step for the implementation of EUV Lithography in high-volume manufacturing. The main challenges for any mask defect inspection platform are resolution, sensitivity, and throughput. The reflective-mode EUV mask scanning lensless imaging microscope (RESCAN) is being developed to provide actinic patterned mask inspection capabilities for defects and patterns with high resolution and high throughput, for node 7 and beyond. Namely, the first goal of the RESCAN project is to develop a tool capable of inspecting an EUV reticle in about 7 hours and detect mask defects down to a size of $10 \mathrm{~nm}$. The lensless imaging concept allows to overcome the resolution limitations due to the numerical aperture (NA) and lens aberrations of conventional actinic mask imaging systems. With the increasing availability of computational power and the refinement of iterative phase reconstruction algorithms, lensless imaging became a powerful tool to synthesize the complex amplitude of the actinic aerial image providing us also with extremely valuable information about phase and mask 3D effects. Here, we present a brief description of the current prototype of the RESCAN platform and illustrate a few experimental examples of programmed defect detection.
\end{abstract}

Keywords: Actinic, EUV, defects, inspection, reticle, metrology.

\section{INTRODUCTION}

RESCAN is a lensless EUV microscope that is being developed at the Paul Scherrer Institut, to provide actinic mask inspection capabilities for defects and patterns with high resolution and high throughput for node 7 and beyond. Defect detection in RESCAN is based on Scanning Scattering Contrast Microscopy (SSCM) ${ }^{1}$, a concept that allows a fast inspection of EUV reticles with arbitrary patterns and makes it possible to detect and classify different types of defects. In SSCM, we compare the measured scattering signal with a reference diffraction pattern to locate defects and extract some of their characteristics. For precise defect localization, it is necessary to perform an additional computational step to improve the resolution of the defect map: using Scanning Coherent Diffraction Imaging (SCDI) ${ }^{2}$, we can reconstruct the complex amplitude of a high-NA and aberration-free aerial image of the defect. SCDI has already been successfully employed in the context of reticle imaging ${ }^{3,4}$, and we demonstrated a successful implementation of this technique at EUV previously ${ }^{2}$.

Despite all the desirable characteristics of SCDI, up to now, there have been two main obstacles for its industrial implementation for EUV reticle defect inspection: the availability of a suitable EUV coherent source and the lack of a fast EUV detector with high dynamic range. Spatial and temporal coherence are necessary requirements for both defect detection and image reconstruction, while a high flux ensures the necessary throughput to perform the full reticle scan in a reasonable amount of time. High-harmonic sources are remarkably compact and provide a highly monochromatic beam. Nevertheless, despite the recent discoveries in this field ${ }^{5}$, the flux remains orders of magnitude below the desired level. Currently, RESCAN is installed at the Swiss Light Source (SLS), a synchrotron facility within the Paul Scherrer Institut. This solution has the advantage of providing a very high flux and a coherent beam but makes it impractical to operate the tool in a fab. A potential solution for this issue is the development of COSAMI, a new standalone EUV coherent source. COSAMI is now in the technical design phase at PSI and its main specifications are summarized in Table 1. The other requirement to meet for a high-throughput defect inspection system is a detector with sufficient speed and dynamic range, which is also under development.

Extreme Ultraviolet (EUV) Lithography VIII, edited by Eric M. Panning, Kenneth A. Goldberg, Proc. of SPIE Vol. 10143, 101431O · C 2017 SPIE · CCC code: 0277-786X/17/\$18 · doi: 10.1117/12.2258086 
Table 1. Technical specifications for the COSAMI EUV source.

\begin{tabular}{|l|l|}
\hline Undulator radiation wavelength & $13.5 \mathrm{~nm}$ \\
\hline Flux & $1.4 \cdot 10^{15}$ photons $/ \mathrm{s}$ \\
\hline Brilliance & $1.8 \cdot 10^{18}$ photons $/ \mathrm{s} / \mathrm{mm}^{2} / \mathrm{mrad}^{2} / 0.1 \% \mathrm{BW}$ \\
\hline Coherent fraction & $6 \%$ \\
\hline Intensity stability & $10^{-3}$ \\
\hline Device footprint & $12 \mathrm{~m} \times 5 \mathrm{~m}$ \\
\hline
\end{tabular}

Considering an inspection area of about $100 \times 100 \mathrm{~mm}^{2}$, an illumination probe with a size on the sample of $30 \mu \mathrm{m}$ and an overlap between the probes of $40 \%$ (see Figure 1), it would take about $4.4 \cdot 10^{7}$ diffraction patterns to cover the whole sample. The frame rate required to collect all the required images in 7 hours is $1.8 \mathrm{kHz}$.

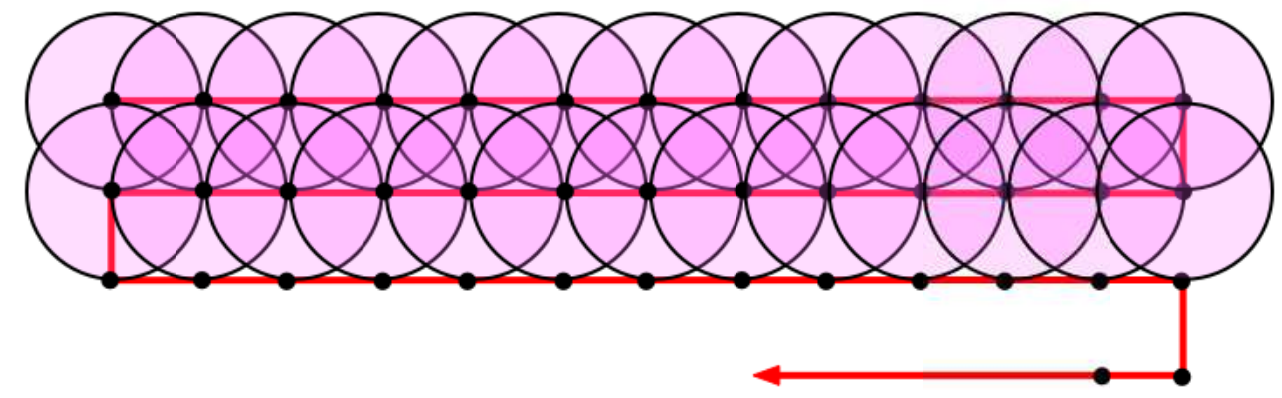

Figure 1. Meander scan for the collection of the diffraction patterns across the sample. The illumination beam (probe) hits the reticle surface at $6^{\circ}$ with a footprint of customizable radius. It is important to cover each portion of the reticle multiple times. The detection algorithm (SSCM) gains in terms of signal to noise ratio and the SCDI step needs the data redundancy to reconstruct the complex amplitude of the reticle image.

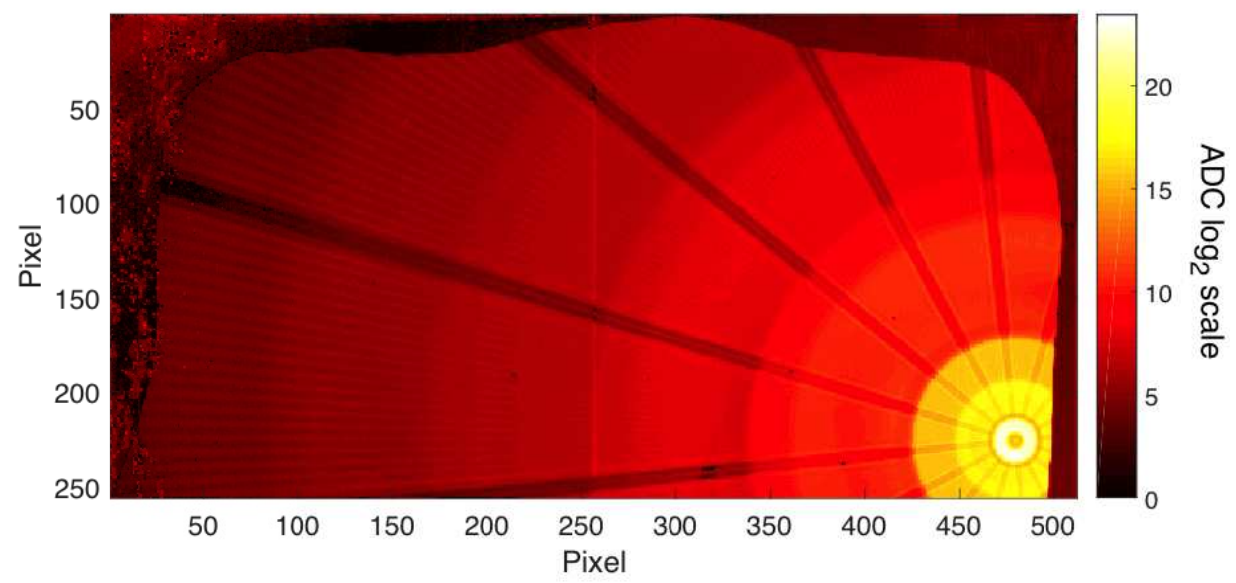

Figure 2. A far-field diffraction pattern of a Fresnel zone plate illuminated at $13.5 \mathrm{~nm}$. The zone plate's diffraction orders have a wide range of divergence angles and create a pattern with a large but discretized intensity variation over a small region. This figure shows one quadrant of a single JUNGFRAU module $(19 \mathrm{~mm} \times 38 \mathrm{~mm})$. The ADC reading is represented on a logarithmic scale to highlight the dynamic range of the detector.

Among the few image detectors that match this specification, we selected a CMOS-based pixel detector originally developed at PSI for free-electron laser applications: The JUNGFRAU ${ }^{6}$ (adJUstiNg Gain detector FoR the Aramis User 
station), is a two-dimensional modular detector under development at PSI. Each JUNGFRAU module has $512 \times 1024$ pixels, each $75 \times 75 \mu \mathrm{m}^{2}$ in size and its readout speed is above $2 \mathrm{kHz}$.

In SCDI, we use the diffraction pattern intensity distribution to reconstruct the reticle structure. When the reticle pattern has a high periodicity, the diffraction pattern can have strong-intensity peaks, while the diffraction signal from an isolated 10-nm defect is typically several orders of magnitude lower; while it is possible to record several images of the same diffraction patterns with different illumination intensities and recombine them to synthesize a high-dynamic range image, this approach is time consuming and can introduce artifacts in the diffraction patterns. This leads to the necessity of a detector with a high dynamic range that can record the whole diffraction pattern in a single acquisition. The unique design of JUNGFRAU is ideal to solve this issue: each pixel has three dynamically adjusting capacitive gain stages that result in a total dynamic range larger than 20 bits. Even though the current prototype of the detector is not optimized for EUV, we successfully tested it at our beamline at $13.5 \mathrm{~nm}$ and recorded an image of the diffraction from a Fresnel zone plate to demonstrate its high dynamic range capability (see Figure 2).

\subsection{The RESCAN prototype}

The current RESCAN prototype is installed at Paul Scherrer Institut, on a dedicated branch of the XIL-II beamline at the Swiss Light Source.

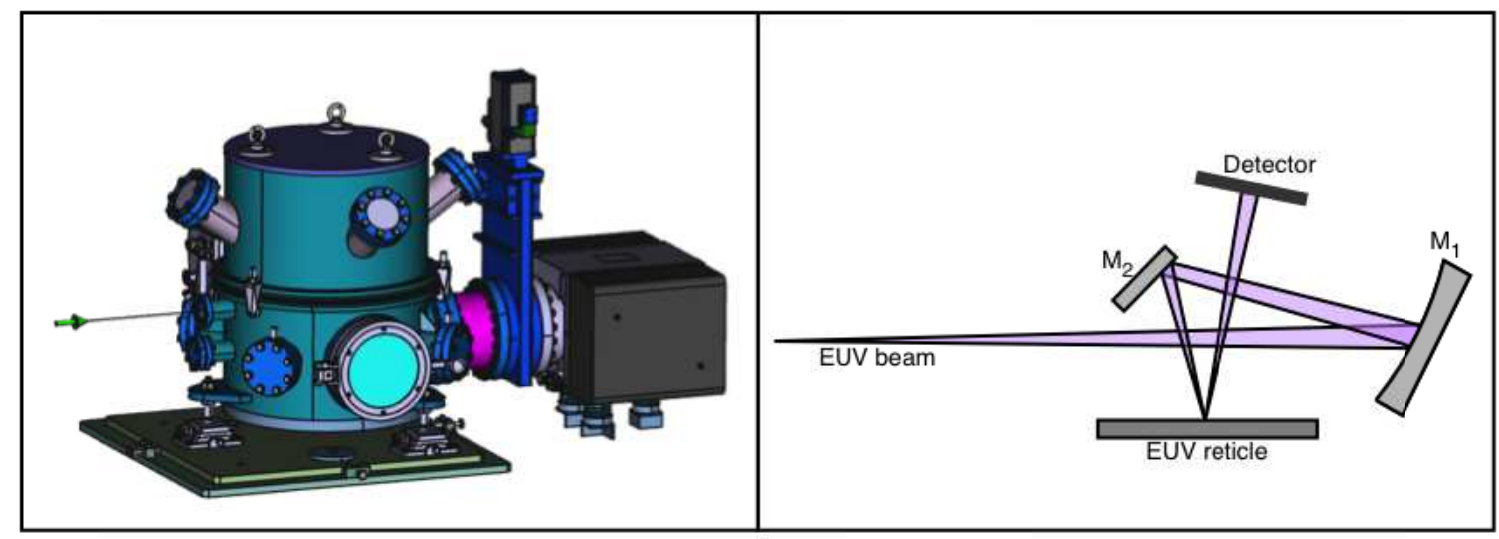

Figure 3.The prototype of the RESCAN platform, operating at the Swiss Light Source. The panel on the left shows a rendering of RESCAN. The optical layout of the tool is shown on the right. The beam from the XIL-II beamline reaches the spherical condenser mirror M1 that focuses it. The beam is folded onto the sample at an AOI of $6^{\circ}$ by the flat mirror M2. The diffraction signal is then recorded by the detector.

RESCAN consists of a compact vacuum chamber connected to the XIL-II beamline (Figure 3 - left panel). A monochromator filters the synchrotron beam to select a bandwidth $\lambda / \Delta \lambda \sim 1600$. The optical system is limited to two optical elements to maximize the photon flux on the sample. The condenser $\left(\mathrm{M}_{1}\right)$, a multilayer-coated spherical mirror with 220 -mm radius, focuses the beam onto a plane close to the sample, and a folding mirror $\left(\mathrm{M}_{2}\right)$ directs the beam towards the sample with an incidence angle of $6^{\circ}$. The current detector is a $2048 \times 2048$ pixel vacuum CCD. The aforementioned JUNGFRAU module will be installed as soon as the EUV optimized version becomes available.

The reticle stage can accommodate samples up to $20 \times 20 \mathrm{~mm}^{2}$ and can scan a maximum area of $200 \times 200 \mu \mathrm{m}^{2}$ with about $1 \mathrm{~nm}$ accuracy.

\section{DEFECT INSPECTION METHOD - OUTLINE}

The defect inspection method we devised for RESCAN consists of two main steps: defect detection, for a fast and coarse assessment of the presence of defects on the reticle, and defect localization, for the precise measurement of the defect position and the evaluation of the defects impact on the aerial image (see Figure 4). Ideally, these two steps can be performed using the same data without affecting the throughput of the method. Both steps rely on the analysis of the diffraction patterns collected as the illumination probe scans different regions of the reticle (cf. Figure 1). 
A

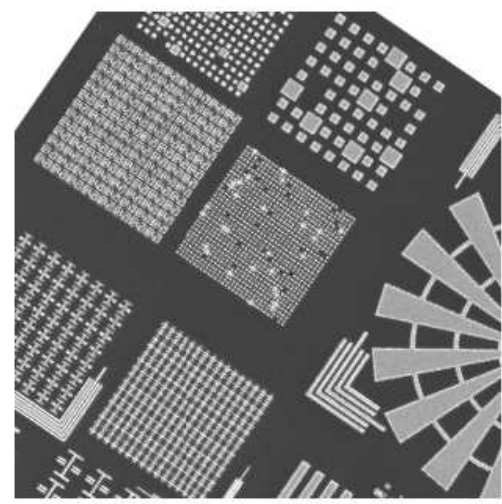

B

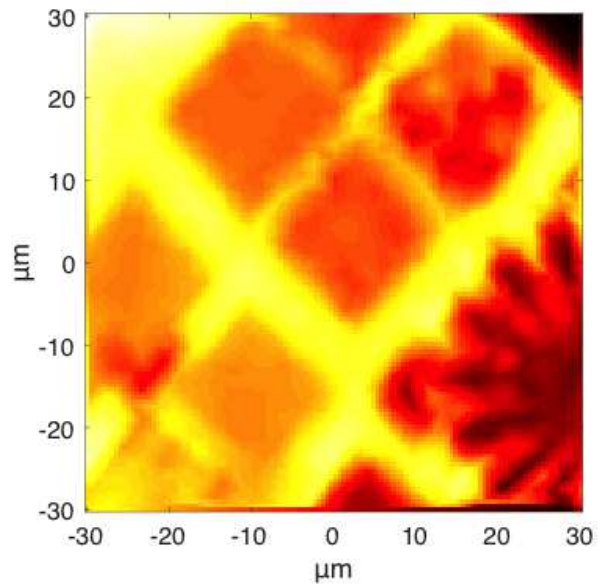

C

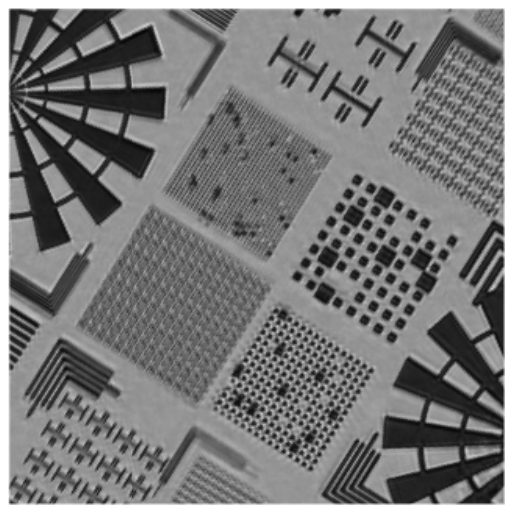

Figure 4. (A). SEM image of a test sample developed at PSI. (B). A low-resolution map, extracted from the zero order of the recorded diffraction patterns. (C) Reticle structure reconstruction performed on a different area of the sample using the diffraction data collected scanning the sample with a $\sim 10-\mu \mathrm{m}$ probe and a $3-\mu \mathrm{m}$ step size.

Considering a minimum probe area overlap of $40 \%$, a reticle area $\mathrm{A}$ of $100 \times 100 \sim \mathrm{mm}^{2}$, a frame acquisition rate $\mathrm{f}=2 \mathrm{kHz}$ and a scanning time $\mathrm{T}$ of 7 hours, the minimum probe radius we can accept is given by:

$$
R=\sqrt{\frac{A}{T f}}=14 \mu m
$$

In the case of a circular spot size, a minimum probe area overlap of $40 \%$ corresponds to a step size of R/2. In RESCAN we can select the illumination NA in discrete steps from 0.002 to 0.02 using different pupil stop apertures. To control the probe size, we change the focal position of our optical system. Probe size and shape metrology are performed with the help of a utility sample with an isolated $2-\mu \mathrm{m}$ contact hole on an EUV reticle scanned with a step size of $1 \mu \mathrm{m}$. We create a map of the reflected beam intensity and deconvolve the contact shape to obtain the probe profile on the sample plane (cf. Figure 4).
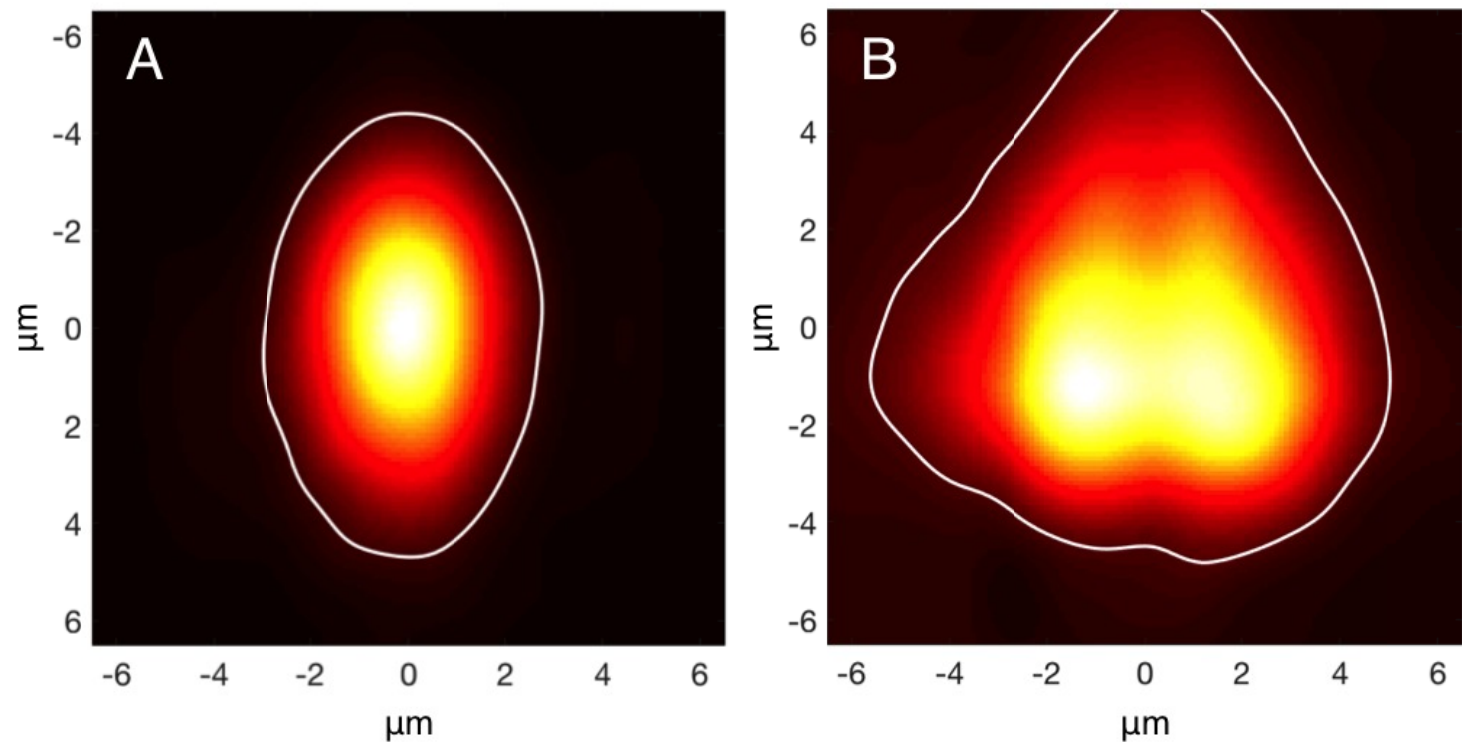

Figure 5. We mapped the footprint of the beam on the sample plane with two condenser NA values using a 2- $\mu \mathrm{m}$ contact hole target. (A) $-\mathrm{NA}=0.0023$. (B) $-\mathrm{NA}=0.0068$. The white contour marks the $10 \%$ intensity threshold and we used it as a reference to define the boundaries of the probe. 
RESCAN's condenser mirror works off-axis with an AOI of $4^{\circ}$. For this reason and because of the intrinsic aberrations of the beamline, the illumination probe has a distinctive astigmatic behavior. Therefore, applying the correct amount of defocus, we can obtain an elongated probe. By scanning the sample in the direction of the elongation, we can maximize the probe overlap to optimize the performance of the defect inspection algorithms.

\subsection{Defect localization}

Once a defect has been detected, it is necessary to perform a precise measurement of its position and characteristics. For this purpose, we use SCDI. We developed a set of algorithms based on ptychography ${ }^{7}$ to reconstruct the complex amplitude of a high-NA and aberration-free aerial image of the defect's area. We can then find the position of the defect with respect to the mask pattern with an accuracy of one nanometer. The reconstruction algorithm is an iterative process:

1. Generate an initial guess of the mask to be reconstructed and of the illumination probe's complex amplitude.

2. Propagate the complex amplitude to the detector plane.

3. Compare the recorded diffraction pattern to the one obtained from the propagation calculation.

4. Adjust the probe function and the mask function to minimize the difference obtained in the previous step.

5. Propagate back to the reticle plane.

Thanks to the information redundancy generated by the overlapping of the probes, the algorithm converges to a solution from which we can extract the phase and amplitude of the mask pattern and of the illumination probe by iterating steps 2 to 5. Although this algorithm is relatively noise-robust, it is sensitive to probe position errors and sample vibrations. For this reason, a major effort was made to optimize the general algorithm for maximum performance in RESCAN.

\subsection{Defect detection}

The first step in our defect inspection approach is to perform a relatively quick detection of the defects present on an EUV reticle. The technique we developed to achieve this task is SSCM. The concept consists in the comparison of the diffraction pattern collected illuminating the reticle and a reference diffraction pattern either calculated with rigorous diffraction simulation of a defect-free mask (die to database) or recorded from a reference reticle (die to die).

Let the complex amplitude of the illumination probe on the sample plane be $\psi(x, y)$, and let the mask reflectivity function be $M(x, y,)_{\lambda, \theta}$, where $\lambda$ and $\theta$ are the wavelength and the angle of incidence. The diffraction pattern intensity of the defect-free mask, $I_{\mathrm{R}}$, can be expressed as:

$$
I_{R}\left(\xi_{x}, \xi_{y}\right)=|\mathcal{F}(M(x, y) \psi(x, y))|^{2} .
$$

In presence of a defect, the mask transmission function is altered and the new diffraction pattern intensity on the detector plane becomes:

$$
I\left(\xi_{x}, \xi_{y}\right)=|\mathcal{F}((M(x, y)+\varepsilon(x, y)) \psi(x, y))|^{2}
$$

Because of the linearity of the Fourier transform, Equation 3 can be expanded into:

$$
I\left(\xi_{x}, \xi_{y}\right)=|\mathcal{F}(M(x, y) \psi(x, y))+\mathcal{F}(\varepsilon(x, y) \psi(x, y))|^{2} .
$$

Let $\mathcal{F}(M(x, y) \psi(x, y))=A e^{i \alpha}$ and $\mathcal{F}(\varepsilon(x, y) \psi(x, y))=B e^{i \beta}$, then Equation 4 becomes:

$$
I\left(\xi_{x}, \xi_{y}\right)=A^{2}+2 A B \cos (\alpha-\beta)+B^{2} .
$$

The difference between a diffraction pattern measured in the presence of a defect and the reference one is then given by:

$$
I-I_{R}=2 A B \cos (\alpha-\beta)+B^{2},
$$

where $B^{2}$ is the diffraction intensity of the defect, and the cross-product is an interference term. The diffraction from the mask pattern usually exhibits strong peaks because of the mask periodicity whereas small and isolated defects have a low diffraction intensity. Therefore, we get most of the difference signal from the interference between the diffraction of the mask and the diffraction of the defects. To maximize the signal-to-noise ratio for defect detection, we use the a-priori 
knowledge of the mask pattern to calculate a map of the diffraction intensity where we can expect maximum contrast for the defect signal. This method has been thoroughly tested in simulation by verifying its performance in presence of Poisson noise and different amounts of line edge roughness ${ }^{8}$.

\section{PROGRAMMED DEFECT INSPECTION}

To test the capabilities of RESCAN experimentally, we manufactured several samples with different features and characteristics. In particular, we included a set of different programmed defects. We patterned 1:1 gratings with different orientation on a silicon wafer coated with a Mo-Si multilayer designed for $6^{\circ}$ incidence at $13.5 \mathrm{~nm}$. The absorber consists of a 40-nm thick Nickel layer, patterned by a lift-off process (see Figure 6).

\begin{tabular}{|l|l|}
\hline $200 \mathrm{~nm}$ & $\begin{array}{l}\text { Figure 6. Top-down SEM image of a } \\
\text { horizontal grating with 100-nm half } \\
\text { pitch defect of 100×100 nm. The } \\
\text { sample consists of a silicon wafer with } \\
\text { a Mo-Si multilayer designed for } \\
\text { maximum reflectivity at 13.5 nm and } \\
6^{\circ} \text { AOI. The absorber is a } 40-\mathrm{nm} \\
\text { nickel layer patterned with a lift-off } \\
\text { process. The visual quality of the } \\
\text { sample is rather poor in terms of line } \\
\text { edge roughness, and the SEM } \\
\text { inspection of a random portion of the } \\
\text { pattern revealed several smaller non- } \\
\text { programmed defects. }\end{array}$ \\
\hline
\end{tabular}
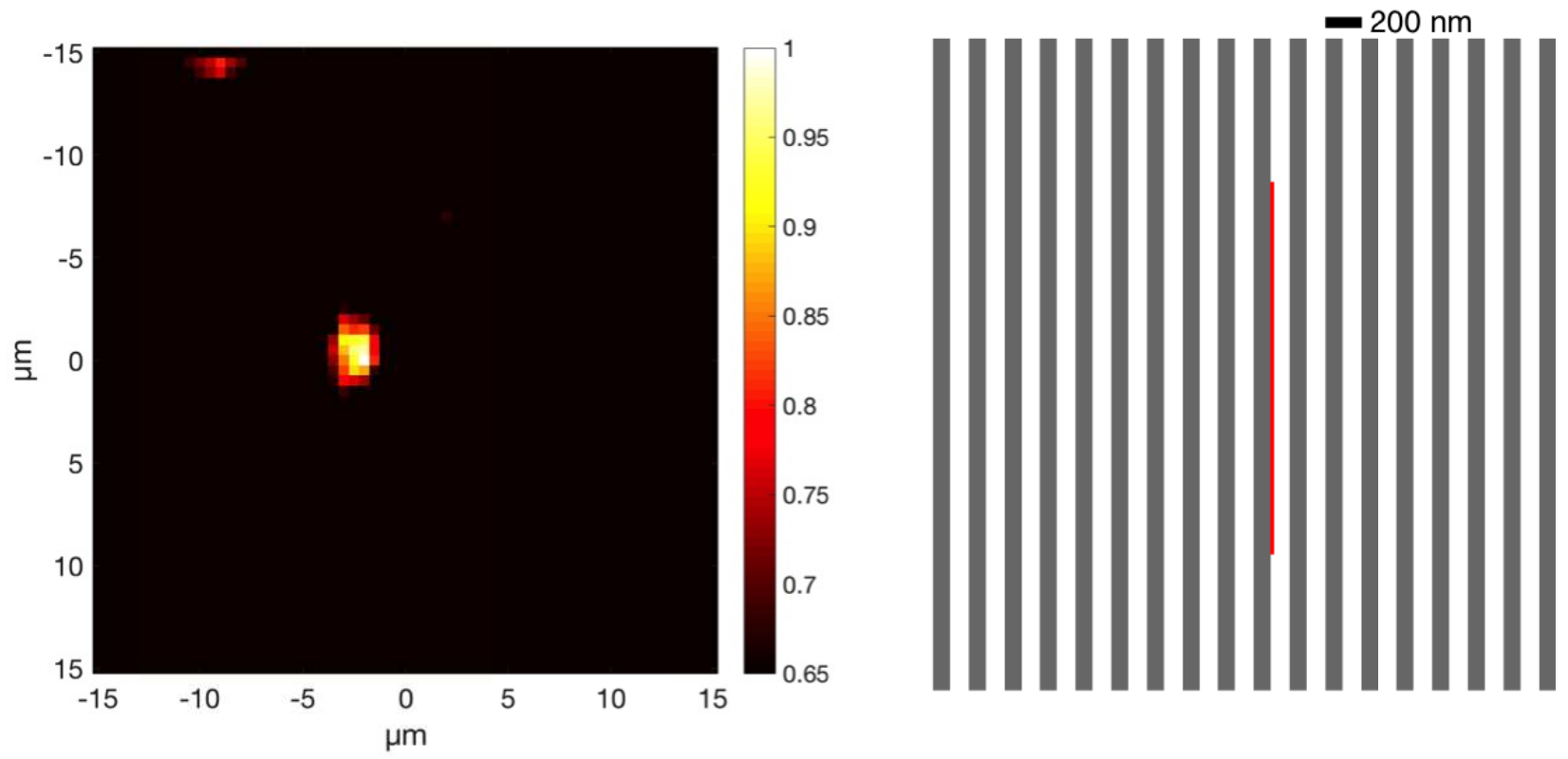

Figure 7. The left picture shows the defect map obtained running an inspection scan over a grating sample with 100-nm CD and a programmed $\mathrm{CD}$ error of $10 \mathrm{~nm} \times 5 \mu \mathrm{m}$. The signal to noise ratio in this case was 6.9. The right picture shows the layout of the sample with the CD error defect highlighted in red. This layout, repeated periodically over an area of $2 \times 2$ $\mathrm{mm}^{2}$, was patterned on a multilayer mirror topped with 40-nm nickel.

Currently, the standard absorber stack for EUV reticles consists of 70-nm TaN with a 2-nm Ru capping layer, but other materials are being investigated as possible absorber candidates to reduce mask 3-D effects. Among them, Nickel seems 
to be one of the more promising ones ${ }^{9}$ and, for us, it has the advantage to be a material that we can process in house at PSI.

The main grating's pitch is $100 \mathrm{~nm}$ (in mask dimensions) and we included two types of programmed defects: a CD error that consists of a $10-\mathrm{nm}$ line extrusion over a length of $5 \mu \mathrm{m}$ (see Figure 7), and a $100 \times 100-\mathrm{nm}^{2}$ bridge defect (see Figure 8 ). We used a $5-\mu \mathrm{m}$ probe with $0.002 \mathrm{NA}$ and scanned an area of $30 \times 30 \mu \mathrm{m}^{2}$ around the expected defect location with a step size of $2 \mu \mathrm{m}$. Despite the grating's line edge roughness, visible in the SEM picture shown in Figure 6, in both cases the programmed defect was detected with a high signal to noise ratio of 6.9 and 7.1 for the for the CD error and the bridge defect, respectively.
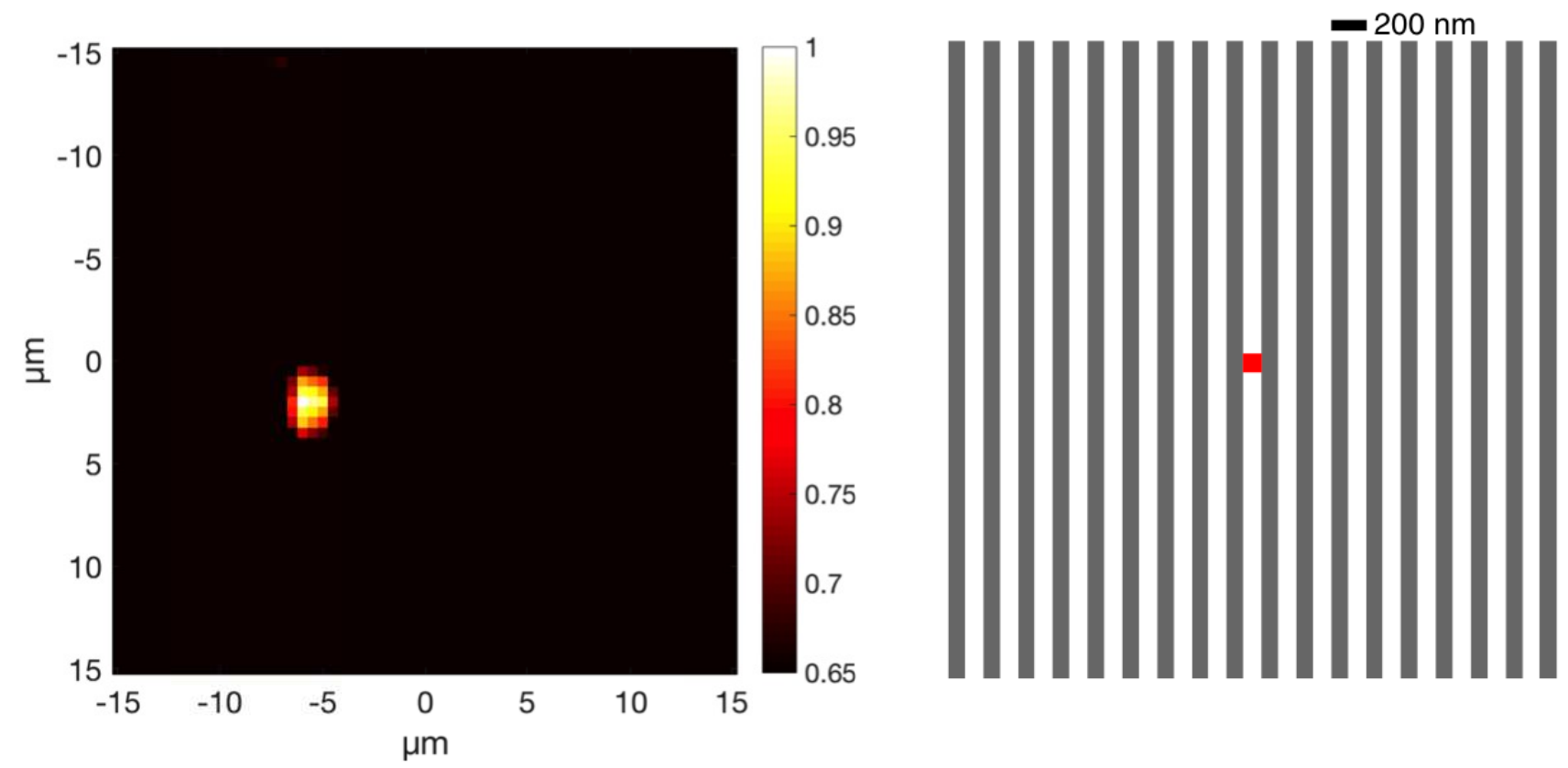

Figure 8. The left picture shows the defect map obtained running an inspection scan over a grating sample with 100-nm CD and a 100-nm programmed bridge defect. The signal to noise ratio in this case was 7.1. The right picture shows the layout of the sample with the bridge defect highlighted in red. This layout, repeated periodically over an area of $2 \times 2 \mathrm{~mm}^{2}$, was patterned on a multilayer mirror topped with $40-\mathrm{nm}$ nickel layer. See figure 5 .

\section{CONCLUSIONS AND OUTLOOK}

RESCAN is an actinic pattern inspection microscope under development at PSI. It a possible candidate to fill the gap in the mask metrology infrastructure required for the implementation of EUV lithography in high-volume manufacturing. The microscope is based on a lensless inspection and imaging approach that makes it easily scalable for high NA and future technology nodes. Currently, a working prototype of the microscope is installed at Paul Scherrer Institut on a dedicated branch of the XIL-II beamline at the Swiss Light Source. We reported here the latest programmed defect inspection results including a $100 \times 100-\mathrm{nm}^{2}$ bridge defect and a $10-\mathrm{nm}$ CD error on a $100-\mathrm{nm}$ half pitch grating. Both defects were detected with a high SNR despite the high line edge roughness of the features on the test samples. We note that the achieved SNR was limited by very high diffused scattering due to the line edge roughness. We plan to keep developing and testing RESCAN with native and programmed defect samples to assess the limits of its performance.

The key elements to make RESCAN a viable candidate for industrial application are a compact, high-intensity, coherent source and an EUV detector with high frame rate and high dynamic range. These two components are the COSAMI source, currently in the technical design stage at PSI, and the JUNGFRAU detector which we are already testing on our microscope. The latter is going to be the next upgrade to RESCAN and will enable it to achieve the scanning speed required for full reticle inspection. We believe that we have a clear path towards the realization of a stand-alone actinic patterned mask inspection tool with targeted performance specifications. 


\section{REFERENCES}

[1] Mohacsi, I., Helfenstein, P., Rajendran, R.., Ekinci, Y., "Scanning scattering contrast microscopy for actinic EUV mask inspection," 2016, 977810 - 977810 - 11.

[2] Helfenstein, P., Mohacsi, I., Rajendran, R.., Ekinci, Y., "Scanning coherent diffractive imaging methods for actinic EUV mask metrology,”2016, 97761F - 97761F - 6.

[3] Harada, T., Hashimoto, H., Amano, T., Kinoshita, H.., Watanabe, T., "Phase imaging results of phase defect using micro-coherent extreme ultraviolet scatterometry microscope," J. MicroNanolithography MEMS MOEMS 15(2), 021007-021007 (2016).

[4] Zhang, B., Adams, D. E., Seaberg, M. D., Gardner, D. F., Shanblatt, E. R., Kapteyn, H.., Murnane, M., "Quantitative tabletop coherent diffraction imaging microscope for EUV lithography mask inspection," 2014, 90501D - 90501D - 9 .

[5] Popmintchev, D., Hernández-García, C., Dollar, F., Mancuso, C., Pérez-Hernández, J. A., Chen, M.-C., Hankla, A., Gao, X., Shim, B., et al., "Ultraviolet surprise: Efficient soft x-ray high-harmonic generation in multiply ionized plasmas," Science 350(6265), 1225 (2015).

[6] Redford, S., Bergamaschi, A.., Brückner, M., "Calibration status and plans for the charge integrating JUNGFRAU pixel detector for SwissFEL," J Inst 11(11), C11013-C11013 (2016).

[7] Thibault, P., Dierolf, M., Bunk, O., Menzel, A.., Pfeiffer, F., "Probe retrieval in ptychographic coherent diffractive imaging," Ultramicroscopy 109(4), 338-343 (2009).

[8] Ekinci, Y., Helfenstein, P., Rajeev, R., Mochi, I., Mohacsi, I., Gobrecht, J.., Yoshitake, S., "Scanning coherent scattering methods for actinic EUV mask inspection," SPIE Photomask Technol., B. S. Kasprowicz and P. D. Buck, Eds., 99851P - 9, SPIE (2016).

[9] Woo, D. G., Kim, J. H., Kim, J. S., Hong, S.., Ahn, J., "Study of Novel EUV Absorber : Nickel \& Nickel Oxide," Korean J Met Mater 55(3), 198-201 (2017). 